\title{
Summary Measures of Adherence Using Pill Counts in Two HIV Prevention Trials: The Need for Standardisation in Reporting
}

\author{
Kathy Baisley $\cdot$ Jared M. Baeten $\cdot$ James P. Hughes $\cdot$ \\ Deborah J. Donnell $\cdot$ Jing Wang $\cdot$ Richard Hayes • \\ Deborah Watson Jones · Connie Celum
}

Published online: 26 June 2013

(C) The Author(s) 2013. This article is published with open access at Springerlink.com

\begin{abstract}
For trials of user-dependent HIV prevention products, accurate adherence measurements are essential to interpret and compare results across trials. We used pill count data from two recent HIV prevention trials of herpes simplex virus type 2 (HSV-2) suppression, to show that estimates of adherence vary substantially depending on assumptions that are made in analysing pill count data. We associate calculated adherence with biological markers of anti-HSV-2 activity. In both trials, calculated adherence varied considerably, depending on the summary measure used, and the handling of intervals with apparent 'overadherence' (fewer pills returned than expected), and unreturned pills. Intervals of apparent over-adherence were associated with reduced antiviral effects on biological markers of herpes reactivation, indicating these are likely to represent periods of non-adherence. Our results
\end{abstract}

K. Baisley $(\varangle) \cdot$ R. Hayes

Department of Infectious Disease Epidemiology, London School of Hygiene and Tropical Medicine, London, UK

e-mail: kathy.baisley@1shtm.ac.uk

J. M. Baeten · D. J. Donnell · C. Celum

Department of Global Health, University of Washington,

Seattle, WA, USA

J. M. Baeten · C. Celum

Department of Medicine, University of Washington, Seattle,

WA, USA

J. M. Baeten · C. Celum

Department of Epidemiology, University of Washington,

Seattle, WA, USA

\section{J. P. Hughes}

Department of Biostatistics, University of Washington,

Seattle, WA, USA demonstrate the clear need for standardisation in reporting of adherence data that are based on pill counts.

Keywords Adherence $\cdot$ HIV prevention - Pill counts

\section{Introduction}

Novel, user-dependent, biomedical HIV prevention interventions require high adherence to achieve efficacy, as demonstrated in clinical trials of tenofovir gel $[1,2]$, daily oral antiretroviral (ARV) pre-exposure prophylaxis (PrEP) [2-6], and ARVs taken by HIV-positive persons to prevent transmission [7]. For such user-dependent methods, with dosing either at fixed intervals or linked to specific events (such as sex acts), accurate and objective measurements of adherence are critical for understanding trial results, since

\section{J. Donnell}

Vaccine and Infectious Disease Division, Fred Hutchinson

Cancer Research Center, Seattle, WA, USA

\section{J. Wang}

Statistical Center for HIV/AIDS Research \& Prevention

(SCHARP), Fred Hutchinson Cancer Research Center,

Seattle, WA, USA

D. W. Jones

Department of Clinical Research, London School of Hygiene and Tropical Medicine, London, UK

D. W. Jones

Mwanza Intervention Trials Unit, National Institute

for Medical Research, Mwanza, Tanzania 
limited or zero effectiveness of an investigational product may be due to either the product's lack of biological efficacy or sub-optimal user adherence [8-10]. Moreover, correlating the level of HIV protection to the level of adherence might provide valuable insight into the relationship between a product's pharmacokinetic properties and its biologic activity $[6,11,12]$. Lastly, understanding patterns and correlates of adherence can inform the design of future prevention trials of similar interventions by providing realistic estimates of possible effect sizes based on achievable levels of adherence.

Various methods have been used to gather information on adherence in biomedical HIV prevention trials, including self-report by face-to-face $[3,6,13]$ or computerassisted [13] interview, participant diaries [14], drug dispensing records [3], electronic monitoring of dosing [4], drug levels in blood, urine or tissues $[1,3,6]$ and biological markers, such as HIV plasma viral load in studies of ARVs to reduce HIV transmission [4]. The simplest and most commonly used measure for quantifying adherence to HIV prevention interventions has been to count unused study product (for example, remaining pills in trials of oral PrEP or unused applicators in microbicide trials) returned at scheduled study visits.

A challenge to interpreting and comparing trial results is the lack of standardization in defining, measuring, analysing and reporting adherence for HIV prevention trials. Trials often report simple summary measures of adherence, with heterogeneity in calculation of these summary measures [15-18]. The absence of a common reporting standard undermines interpretation of adherence patterns, product effectiveness, and predictors of adherence. Even with seemingly simple methods such as pill counts, adherence measurement is complex with issues concerning how to handle visits when pills are not returned, apparent 'over-adherence' ( $>100 \%$ of expected pills for the interval between visits), missed visits (when product is thus not dispensed for a period) and protocol-specified time off treatment such as during pregnancy.

An additional challenge is the lack of a standardised adherence terminology with clear definitions to designate the same concepts across trials. Recent reviews have proposed a new taxonomy for adherence research, with uniform terms and definitions [19, 20]. 'Adherence to medication' describes the participant's use of the study product as prescribed. Adherence has three components: initiation (the time point of first dose), execution (the extent to which the participant's product use corresponds to the instructed regimen), and discontinuation (the time point when the participant stops using the product). A fourth term, persistence, describes the length of time between initiation and discontinuation.

We propose an additional term, adherence 'coverage', to describe a participant's tablet taking during the entire time the participant is in a trial, including temporary treatment interruptions. This is to recognise that, although the participant may have been instructed to stop taking the drug (and is thus taking the product as prescribed), these periods should be included when reporting trial adherence. Adherence coverage is arguably the most relevant measure for interpreting trial results, as it provides an indication of whether sufficient study medication was taken to expect a biological response. We also propose the term 'apparent over-adherence' to describe periods when counts of returned study product suggest that the participant has taken $>100 \%$ of expected doses.

In this paper, we use data from two recent biomedical HIV prevention trials of herpes simplex virus type 2 (HSV2) suppression, which both dispensed pills to be taken twice daily and used clinic-based pill counts of returned study product to measure adherence, to demonstrate how estimates of adherence vary depending on the assumptions that are made in analysing and reporting adherence data. In addition, we relate calculated adherence to biological markers of pill activity (genital herpes reactivation), to assess the value of pill counts as a measure of 'true' adherence.

\section{Methods}

We used data from two double blind, placebo-controlled trials of daily HSV-2 suppressive therapy with acyclovir for HIV prevention: one in Mwanza, Tanzania [21] and HPTN 039, which was conducted in nine sites in Peru, South Africa, United States, Zambia, and Zimbabwe[22] (Table 1). Participants were randomised to twice daily acyclovir $400 \mathrm{mg}$ or matching placebo. In both trials, there was no evidence of a difference in HIV incidence between the acyclovir and placebo arms overall, whilst among the sub-group with optimal adherence, those randomized to acyclovir had decreased HSV-2 activity (genital ulcers or HSV-2 DNA shedding) compared with those randomized to placebo $[21,22]$. There was no evidence that adherence differed between randomization arms in either trial [22, 23]. In Mwanza, there was no evidence that participants knew their randomization assignment [24]. In HPTN 039, among the one-third of participants who thought that they knew their randomisation assignment in an assessment after the end of the study, a slightly higher proportion of participants in the acyclovir arm than in placebo perceived they were randomised to acyclovir [23].

\section{Mwanza Trial}

The Mwanza trial enrolled 1305 HSV-2 seropositive women aged 16-35 years working in bars, guesthouses and 
Table 1 Summary of two herpes suppression trials for HIV prevention: Mwanza and HPTN 039

\begin{tabular}{|c|c|c|}
\hline & Mwanza trial & HPTN 039 \\
\hline \multicolumn{3}{|l|}{ Study design } \\
\hline Location & Tanzania & $\begin{array}{l}\text { Peru, South Africa, USA, Zambia, } \\
\text { Zimbabwe }\end{array}$ \\
\hline Population & HIV negative and HIV positive women & $\begin{array}{l}\text { HIV negative women and men who have } \\
\text { sex with men }\end{array}$ \\
\hline Number randomised & 1,305 & 3,277 (1395 women, 1882 men) \\
\hline Length of follow up & Up to 30 months & Up to 18 months \\
\hline Frequency of scheduled visits & 3 monthly & Monthly \\
\hline $\begin{array}{l}\text { Could resume tablets after treatment } \\
\text { interruption }\end{array}$ & $\begin{array}{l}\text { Yes, but not for pregnancy or if } \\
\text { participant requested to stop taking the } \\
\text { drug }\end{array}$ & Yes \\
\hline Main endpoints & $\begin{array}{l}\text { HIV incidence; genital and plasma HIV } \\
\text { RNA in HIV/HSV-2 co-infected women }\end{array}$ & $\begin{array}{l}\text { HIV incidence; incidence of HSV-2 } \\
\text { ulcers }\end{array}$ \\
\hline \multicolumn{3}{|l|}{$\begin{array}{l}\text { Adherence measurement for main trial } \\
\text { publications }\end{array}$} \\
\hline Medication dispensed & Blister strips of 14 tablets each & Bottles of 70 \\
\hline $\begin{array}{l}\text { Identification numbers on bottles/ } \\
\text { blister strips }\end{array}$ & $\begin{array}{l}\text { Non-unique batch ID recorded when } \\
\text { dispensed only }\end{array}$ & $\begin{array}{l}\text { Yes, unique ID recorded when dispensed } \\
\text { and returned }\end{array}$ \\
\hline $\begin{array}{l}\text { Amount of surplus tablets ('buffer } \\
\text { stock') supplied at each visit }\end{array}$ & $2-3$ weeks & 5 days \\
\hline \multicolumn{3}{|l|}{ Pill counts } \\
\hline Frequency & Every visit & Every visit \\
\hline Performed by & Dispensing clinician & Dispensing clinician \\
\hline \multicolumn{3}{|l|}{ Self report } \\
\hline Frequency & Scheduled visits & Every visit \\
\hline Questions used & $\begin{array}{l}\text { If missed any tablets since last visit; if } \\
\text { missed } \geq 3 \text { consecutive days of tablets; } \\
\text { total number of tablets missed }\end{array}$ & $\begin{array}{l}\text { If missed any tablets since last visit; if } \\
\text { missed } \geq 2 \text { consecutive doses; } \\
\text { maximum number of consecutive } \\
\text { missed doses }\end{array}$ \\
\hline \multicolumn{3}{|l|}{ Adherence calculations } \\
\hline $\begin{array}{l}\text { Handling of intervals with } \\
\text { adherence }>100 \%\end{array}$ & $\begin{array}{l}\text { Allowed } 1-4 \text { tablets over }{ }^{\mathrm{a}} \text {; if }>4 \text { tablets } \\
\text { over, and did not report taking extra, } \\
\text { classed as missing }\end{array}$ & $\begin{array}{l}\text { Allowed } 5 \% \text { over; if }>5 \% \text { over, classed } \\
\text { as missing }\end{array}$ \\
\hline $\begin{array}{l}\text { Handling of intervals when bottles/ } \\
\text { blister strips not returned }\end{array}$ & Classified as missing & $\begin{array}{l}\text { Asked to return at next visit. If never } \\
\text { returned, used self report. If self-report } \\
<100 \% \text {, classed as missing. }\end{array}$ \\
\hline $\begin{array}{l}\text { Returned pills matched to visit } \\
\text { dispensed }\end{array}$ & $\begin{array}{l}\text { No, assumed to have been dispensed at } \\
\text { previous visit }\end{array}$ & Yes \\
\hline $\begin{array}{l}\text { Handling of intervals when } \\
\text { adherence classed as missing }\end{array}$ & $\begin{array}{l}\text { Replaced as } 70 \% \text { of expected tablets } \\
\text { taken, or all tablets dispensed, } \\
\text { whichever was less }\end{array}$ & $\begin{array}{l}\text { Dropped from numerator and } \\
\text { denominator }\end{array}$ \\
\hline Included periods off treatment & Yes, except for pregnancy & Yes \\
\hline $\begin{array}{l}\text { Summary measures used and } \\
\text { reported adherence }\end{array}$ & $\begin{array}{l}\text { Median adherence }=90 \% \text {; person-years } \\
\text { with } \geq 90 \% \text { adherence }=52 \% \\
(\text { acyclovir arm) and } 51 \% \text { (placebo) }\end{array}$ & $\begin{array}{l}\text { Median } \% \text { of expected doses } \\
\text { taken }=86 \% ; \text { median } \% \text { of dispensed } \\
\text { drug taken }=94 \% ; \% \text { of quarterly } \\
\text { visits with } \geq 90 \% \text { adherence }=73 \%\end{array}$ \\
\hline \multicolumn{3}{|l|}{$\begin{array}{l}\text { Summary of data included in current } \\
\text { analysis }\end{array}$} \\
\hline Total visits attended & 9,199 & 48,446 \\
\hline Scheduled visits & 9,139 & 47,551 \\
\hline Interim visits & 60 & 895 \\
\hline $\begin{array}{l}\text { Total visits analysed (i.e. excluding } \\
\text { visits after pregnancy) }\end{array}$ & 8,149 & 47,243 \\
\hline
\end{tabular}


Table 1 continued

\begin{tabular}{|c|c|c|}
\hline & Mwanza trial & HPTN 039 \\
\hline $\begin{array}{l}\text { Participants with at least one } \\
\text { pill count }\end{array}$ & 1,242 & 3,140 \\
\hline $\begin{array}{l}\text { Participants completing } \\
\text { follow-up }\end{array}$ & $972(78 \%)$ & $2,428(77 \%)$ \\
\hline Person-years of follow-up & 2,144 & 4,081 \\
\hline $\begin{array}{l}\text { Participants with treatment } \\
\text { interruption }\end{array}$ & $233(19 \%)^{\mathrm{b}} ; 14(1 \%)^{\mathrm{c}}$ & $183(6 \%)^{\mathrm{b}} ; 48(2 \%)^{\mathrm{c}}$ \\
\hline Person-years off treatment & $0.73^{\mathrm{c}}$ & $13^{\mathrm{c}}$ \\
\hline \multicolumn{3}{|l|}{$\begin{array}{l}\text { Visits with 'measurable' } \\
\text { adherence }\end{array}$} \\
\hline $\begin{array}{l}\% \text { visits with tablets } \\
\text { returned on time }\end{array}$ & $95 \%{ }^{\mathrm{d}}$ & $89 \%$ \\
\hline $\begin{array}{l}\% \text { visits with late/ } \\
\text { unscheduled returns }\end{array}$ & $3 \%$ & $7 \%$ \\
\hline \multicolumn{3}{|l|}{$\begin{array}{l}\text { Visits with 'unmeasurable' } \\
\text { adherence (tablets never } \\
\text { returned) }\end{array}$} \\
\hline$\%$ of all visits & $2 \%$ & $4 \%$ \\
\hline$\%$ of participants ever & $12 \%$ & $31 \%$ \\
\hline \multicolumn{3}{|l|}{$\begin{array}{l}\text { Apparent over-adherence } \\
(>105 \%)\end{array}$} \\
\hline$\%$ of all visits & $19 \%$ & $11 \%$ \\
\hline Median (IQR) tablets over & $28(16-46)$ & $6(4-10)$ \\
\hline$\%$ of participants ever & $66 \%$ & $65 \%$ \\
\hline \multicolumn{3}{|c|}{$\begin{array}{l}\text { In the calculations of adherence summary measures for this paper (Methods } 1 \text { and 2), we allowed up to } 5 \% \text { over-adherence in Mwanza instead } \\
\text { of } 1-4 \text { tablets over, for comparability with HPTN } 039\end{array}$} \\
\hline \multicolumn{3}{|c|}{ Participants with treatment interruptions for pregnancy (censored at pregnancy for adherence calculations) } \\
\hline \multicolumn{3}{|c|}{ Participants with treatment interruptions not related to pregnancy } \\
\hline
\end{tabular}

similar facilities in 19 communities in northwest Tanzania. The trial enrolled both HIV negative (821) and HIV positive (484) women, to examine the effect of acyclovir on HIV acquisition and among HIV-infected women, on HIV genital shedding and viral load. Women were followed every 3 months for 12-30 months. Women were withdrawn permanently from the study medication if they became pregnant, and study medication was withdrawn temporarily for medical reasons such as intercurrent illness. Women who were withdrawn from tablets continued to attend follow-up visits, unless they requested to withdraw completely from the trial.

At each quarterly visit, women were issued with a supply of tablets to last until their next scheduled visit, plus an additional 2-3 week buffer stock in case they were late. Tablets were supplied in blister strips of 14 tablets. The batch number was recorded when dispensed, but not when returned. At each visit, women were asked to bring unused tablets and empty blister strips from the preceding visit; the number of remaining tablets was recorded by the dispensing clinician. In addition to pill counts, women were asked about self-reported adherence.

\section{HPTN 039 Trial}

The HPTN 039 trial enrolled 1,358 women and 1,814 men who have sex with men who were HSV-2 seropositive and HIV negative. Participants were followed monthly for 12-18 months with quarterly pregnancy and HIV testing. Women with positive pregnancy tests were withdrawn from study medication until pregnancy tests were negative, and then tablets were resumed. Women who were withdrawn from study medication continued to attend followup.

At each visit, participants were issued with a supply of tablets to last until their next visit, scheduled every 30 days, plus a 5-day buffer stock in case they were late. Tablets were packaged in bottles of 70; each bottle had a unique identification number that was recorded when dispensed and returned. At each visit, participants were asked 
to return the bottle and unused tablets from the preceding month, which were counted. If participants failed to return a bottle, they were asked to return it at their subsequent monthly visit and were instructed not to take any more tablets from it. At each visit, participants were also asked about self-reported adherence.

\section{Adherence Calculations}

We used pill count data from the Mwanza and HPTN 039 trials to analyze adherence 'coverage', defined as tablet-taking during the entire time the participant was in the trial, including missed visits and temporary treatment interruptions.

For each participant, we calculated adherence at each visit as [tablets issued at last visit - tablets returned]/[days elapsed since last visit $\times 2$ (given the twice-daily use of the study tablets)]. For HPTN 039, bottles that were returned late were matched to the visit when they were dispensed. In the Mwanza trial, blister strips could not be matched to the visit when dispensed since that information was not recorded; thus, all returned tablets were assumed to have been dispensed at the previous visit. Adherence was set to 0 during periods of treatment interruption. We defined 'optimal' adherence as taking $\geq 90 \%$ of expected doses in the interval between visits.

For the Mwanza trial, since the primary analysis was modified intent-to-treat that censored women at pregnancy, for the adherence calculations, we excluded visits after women were permanently withdrawn from tablets for pregnancy. In addition, for comparability with Mwanza, we censored women at pregnancy for the adherence calculations in HPTN 039; although the primary intent-to-treat analysis included periods off treatment for pregnancy. Thus, our estimates of adherence coverage exclude visits after pregnancy.

\section{Summary Measures to Describe Adherence During the Trial}

To describe average adherence coverage during the trial, we used two summary measures commonly reported in clinical trials to describe central tendency: overall study adherence and median adherence. Overall study adherence was calculated as [total tablets taken by all participants]/ [total days in study for all participants $\times 2$ ]. Median adherence was calculated in two ways: (1) calculating adherence at each visit for each participant, then computing the median of per-visit adherence over all visits for all participants; and (2) calculating overall adherence for each participant, then computing the median of per-participant adherence.

To describe optimal adherence, we used three measures commonly reported in clinical trials: (1) the proportion of visits with $\geq 90 \%$ adherence; (2) the proportion of person- years with $\geq 90 \%$ adherence; and (3) the proportion of participants with $\geq 90 \%$ adherence during their entire study participation, defined as [total tablets taken]/[total days in study $\times 2] \geq 90 \%$.

\section{Assessment of Missing Data and Over-Adherence}

In trials that use pill counts to assess adherence, participants are asked to return the pill containers (e.g. bottles, blister packs) at each visit so that any remaining tablets can be counted. The underlying assumption is that the remaining tablets reflect the number that were dispensed at the previous visit minus the number that were ingested by the participant. However, generally there will be some visits when the containers are not returned, or when fewer than the expected number of tablets are remaining, and thus adherence is calculated to be $>100 \%$ ('apparent over-adherence'). To calculate a summary measure of adherence, assumptions must be made about the number of tablets taken for those visits. We used two methods to explore the impact of these assumptions on summary measures, drawing on methods used by each trial in their primary publications.

Method 1 was based on the approach used in HPTN 039 [22], allowing adherence up to $105 \%$ for each interval, due to imprecision in being able to ascertain timing of last dose with twice-daily dosing. If pill count data indicated adherence was 100-105\%, we assumed the participant had taken the tablets, and re-set adherence to $100 \%$ for all calculations. However, if pill count data indicated adherence $>105 \%$, or if tablets were not returned, we classified adherence as 'unknown'. Intervals with unknown adherence were removed from the numerator and denominator and excluded from the calculation of summary measures; this approach assumes that the distribution of adherence in intervals when it is unknown is similar to that when it is known.

Method 2 was based on the approach used in the Mwanza trial [21], also allowing up to $5 \%$ over-adherence (re-set to $100 \%$ in calculations) but participants were assumed to have low adherence (70 \%) in intervals when adherence was unknown (tablets not returned or adherence $>105 \%$ ). This approach assumes that intervals with unknown adherence are likely to reflect periods of poor adherence. As a sensitivity analysis, we explored the impact of using a range of adherence levels, from 10 to $100 \%$ (i.e. assuming that all pills were taken), during periods when adherence was unknown.

Use of Biological Marker to Assess Periods with Unknown Adherence

Within each adherence category, we examined the effect of treatment arm on a biological outcome: for HPTN 039, 
genital ulcer disease (GUD) with HSV-2 aetiology confirmed by HSV DNA PCR, based on quarterly genital exams [22], and for Mwanza, genital HSV-2 DNA shedding at the 6,12 and 24 month visits, when samples were collected [21]. Random-effects logistic regression was used to estimate odds ratios (OR) for the association of treatment with the detection of GUD or HSV-2 shedding at each visit. Models contained fixed effects for treatment arm, calculated adherence in the preceding interval, and their interaction, and random effects for subject.

\section{Results}

We analysed 8,149 post-enrolment visits from 1,242 participants in the Mwanza trial and 47,243 visits from 3,140 participants in HPTN 039 (Table 1). In Mwanza, $98 \%$ of blister packs were returned during the trial, and $95 \%$ were returned at the next 3-monthly visit. In HPTN 039, $96 \%$ of bottles were returned during the trial, $89 \%$ at the next monthly visit.

In Mwanza, 247 (20\%) participants interrupted study drug, including 233 who became pregnant and were permanently discontinued from study medication. In HPTN 039, 231 participants interrupted study drug, including 183 who became pregnant (14\% of all females). Time off treatment for non-pregnancy related interruptions was 0.7 person-years in Mwanza and 13 person-years in HPTN 039.

Figure 1 shows the distribution of adherence at each visit based on the actual pill count data. Two-thirds of participants had $\geq 1$ interval when calculated adherence was $>105 \%$, which accounted for 19 and $11 \%$ of visits in Mwanza and HPTN 039, respectively.

In Mwanza, where scheduled visits were quarterly, the proportion of participants with adherence 90-105\% ranged from a low of $46 \%$ at the second visit (month 6) to a high of $57 \%$ at month 18 (Fig. 2). In HPTN 039, with more frequent (monthly) visits, the proportion of participants with adherence $90-105 \%$ ranged from a low of $64 \%$ at the first visit (month 1) to a high of $73 \%$ at month 14 .

In Mwanza, participants self-reported not missing any doses in $19 \%$ of intervals with pill count-based adherence $<75 \%, 6 \%$ of intervals with adherence $75-89 \%, 52 \%$ of intervals with adherence $90-105 \%$, and $84 \%$ of intervals with unknown adherence. In HPTN 039, participants selfreported not missing any doses in $38 \%$ of intervals with adherence $<75 \%, 43 \%$ of intervals with adherence $75-89 \%, 83 \%$ of intervals with adherence $90-105 \%$, and $80 \%$ of intervals with unknown adherence.

\section{Summary Measures of Adherence}

Estimates of average adherence coverage during the trials ranged from 82-98 \% in Mwanza, and 88-100 \% in HPTN
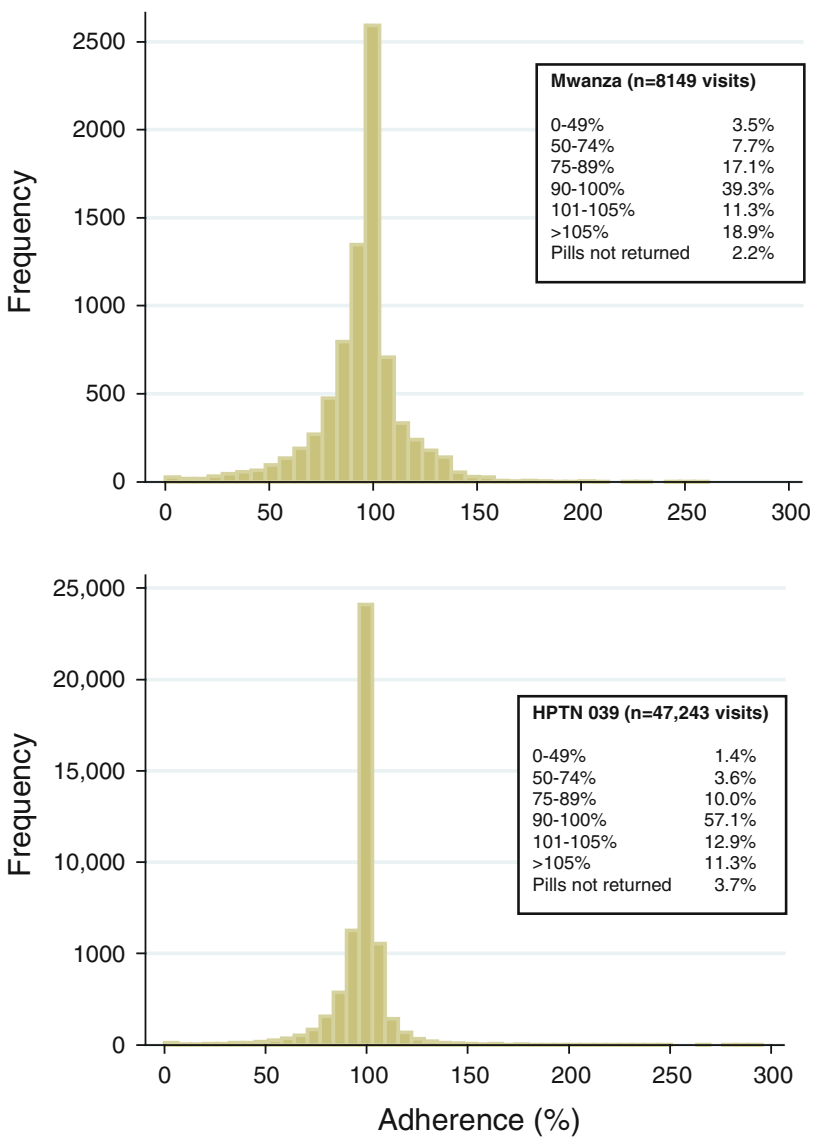

Fig. 1 Estimated adherence at each visit in Mwanza (top) and HPTN 039 (bottom) trials among all participants (including treatment interruptions. In Mwanza, women were permanently withdrawn from study medication if pregnant, but continued with followup; the primary analysis was modified intent-to-treat that censored women at pregnancy. Therefore, for the adherence calculations, visits after women were withdrawn for pregnancy are excluded. In HPTN 039, women were temporarily withdrawn until pregnancy tests were negative, and then tablets were resumed; the primary analysis included periods off treatment for pregnancy. However, for comparability with Mwanza, visits after women were withdrawn for pregnancy are excluded from the adherence calculations and missed visits)

039 , depending on the summary measure and the method used to calculate it (Table 2). Estimates of overall adherence were lower than those of median adherence. Adherence appeared highest when the median per-visit adherence was used; excluding unknown intervals, rather than considering them as periods of poor adherence, made adherence appear higher for all measures. Calculating the median as a per-participant measure gave an impression of lower adherence than calculating median adherence as a per-visit measure. Since median per-visit adherence is based on data at all visits, participants who attend more visits will contribute more information. In contrast, for median per-participant adherence, the data are first aggregated by participant, so all participants contribute equally, regardless of the number of visits attended. 
Fig. 2 Adherence in HPTN 039 and Mwanza trials by pill counts at selected scheduled visits. Adherence in HPTN 039 shown at visits every 3 months, for the preceding month, to show data for each trial at comparable points in follow-up time

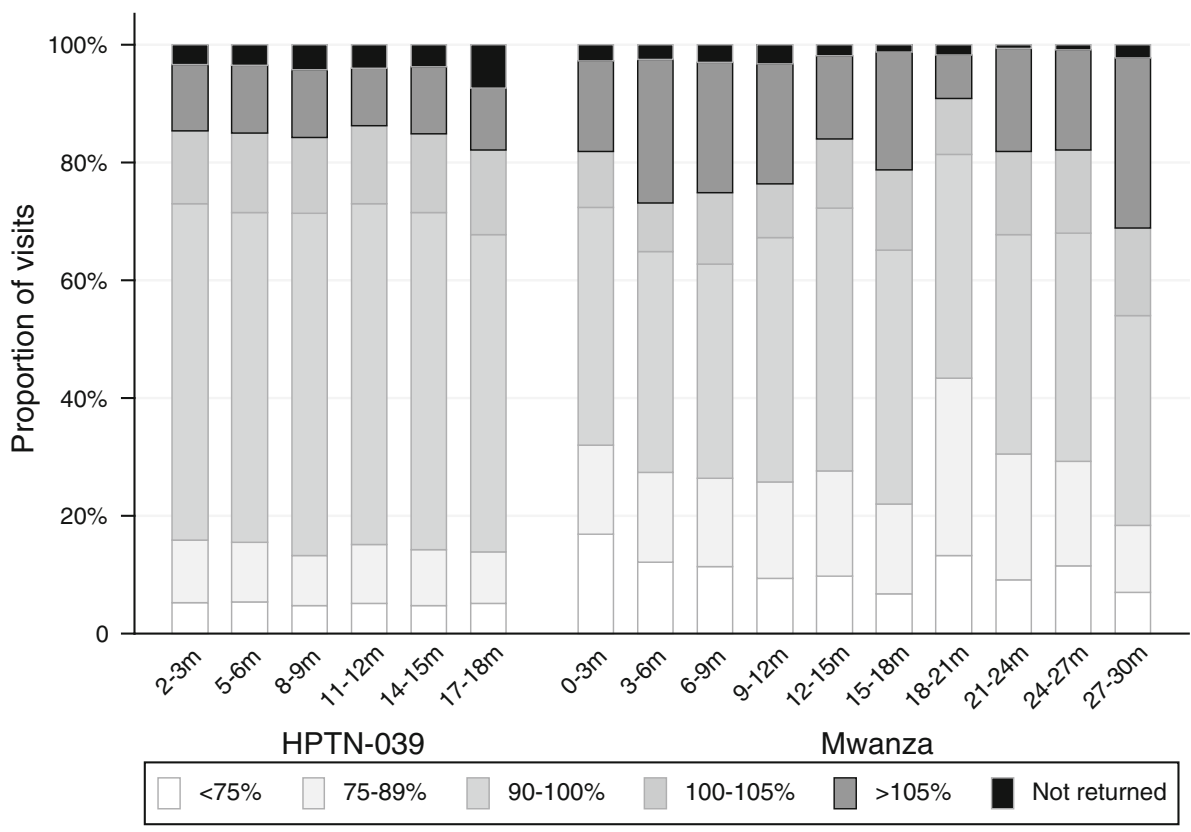

Table 2 Commonly reported summary measures of adherence, with different assumptions regarding intervals with unknown adherence owing to missing tablet counts and apparent over-adherence (>100\%)

\begin{tabular}{|c|c|c|c|c|c|}
\hline \multicolumn{3}{|c|}{ Mwanza trial } & \multicolumn{3}{|l|}{ HPTN 039} \\
\hline \multirow{2}{*}{$\begin{array}{l}\text { Unknown } \\
\text { excluded }^{\text {a }}\end{array}$} & \multirow{2}{*}{$\begin{array}{l}\text { Unknown } \\
\text { replaced } \\
\text { as } 70 \%^{\mathrm{b}}\end{array}$} & Unknown replaced as ${ }^{c}$ & \multirow{2}{*}{$\begin{array}{l}\text { Unknown } \\
\text { excluded }^{\text {a }}\end{array}$} & \multirow{2}{*}{$\begin{array}{l}\text { Unknown } \\
\text { replaced } \\
\text { as } 70 \% \text { b }\end{array}$} & Unknown replaced as ${ }^{c}$ \\
\hline & & $10 \% \quad 30 \% \quad 50 \%$ & & & $10 \% \quad 30 \% \quad 50 \%$ \\
\hline
\end{tabular}

\begin{tabular}{|c|c|c|c|c|c|c|c|c|c|c|c|c|}
\hline \multicolumn{13}{|c|}{ Measures of 'average' adherence coverage } \\
\hline Median per-visit adherence ${ }^{\mathrm{d}}$ & $95 \%$ & $90 \%$ & $90 \%$ & $90 \%$ & $90 \%$ & $98 \%$ & $99 \%$ & $97 \%$ & $97 \%$ & $97 \%$ & $97 \%$ & $100 \%$ \\
\hline $\begin{array}{l}\text { Median per-participant } \\
\text { adherence }\end{array}$ & $89 \%$ & $83 \%$ & $70 \%$ & $74 \%$ & $79 \%$ & $91 \%$ & $95 \%$ & $91 \%$ & $84 \%$ & $86 \%$ & $89 \%$ & $96 \%$ \\
\hline Overall adherence $^{\mathrm{f}}$ & $86 \%$ & $82 \%$ & $71 \%$ & $75 \%$ & $79 \%$ & $88 \%$ & $92 \%$ & $88 \%$ & $80 \%$ & $82 \%$ & $85 \%$ & $91 \%$ \\
\hline \multicolumn{13}{|c|}{ Measures of 'optimal' adherence coverage } \\
\hline $\begin{array}{l}\text { Proportion of visits with } \\
\text { adherence } \geq 90 \%\end{array}$ & $64 \%$ & $51 \%$ & $51 \%$ & $51 \%$ & $51 \%$ & $71 \%$ & $82 \%$ & $70 \%$ & $70 \%$ & $70 \%$ & $70 \%$ & $84 \%$ \\
\hline $\begin{array}{c}\text { Proportion of person years } \\
\text { with adherence } \geq 90 \%\end{array}$ & $61 \%$ & $48 \%$ & $48 \%$ & $48 \%$ & $48 \%$ & $69 \%$ & $79 \%$ & $67 \%$ & $67 \%$ & $67 \%$ & $67 \%$ & $82 \%$ \\
\hline $\begin{array}{l}\text { Proportion of participants } \\
\text { with } \geq 90 \% \text { adherence }\end{array}$ & $48 \%$ & $27 \%$ & $18 \%$ & $19 \%$ & $21 \%$ & $56 \%$ & $73 \%$ & $54 \%$ & $34 \%$ & $39 \%$ & $45 \%$ & $74 \%$ \\
\hline
\end{tabular}

${ }^{a}$ Unknown excluded: allow up to $105 \%$ adherence (re-set to $100 \%$ ), otherwise unknown; exclude intervals with unknown adherence from numerator and denominator (Method 1)

${ }^{\mathrm{b}}$ Unknown replaced as $70 \%$ : allow up to $105 \%$ adherence (re-set to $100 \%$ ), otherwise unknown; assume adherence in unknown intervals is low $(70 \%)($ Method 2)

${ }^{c}$ Sensitivity analysis using a range of adherence levels to replace intervals when adherence is unknown

d Adherence calculated at each visit for each participant as (tablets taken since last visit/days elapsed since last visit $\times 2$ ), then median taken over all visits and participants

e Adherence calculated overall for each participant as (total tables taken/total days in study $\times 2$ ), then median taken over all participants

f Total tablets taken by all participants/total days in study for all participants $\times 2$

$\mathrm{g}$ Adherence for each participant calculated as (total tablets taken during study/total days in study $\times 2$ )

Estimates of optimal adherence $(\geq 90 \%)$ ranged from $27-71 \%$ in Mwanza and 56-84\% in HPTN 039, depending on the method used and whether data were first aggregated by participant before the measure was calculated. Optimal adherence appeared highest when the proportion of visits with adherence $\geq 90 \%$ was used, and unknown intervals were excluded. In contrast, reporting the proportion of participants with adherence $\geq 90 \%$, with 
unknown intervals assumed to represent poor adherence, gave the most pessimistic picture of optimal adherence.

In both trials, participants who attended more visits had higher adherence. In Mwanza, participants' overall adherence during the trial was on average $2.4 \%$ higher for each one-visit increase in total visits attended. In HPTN 039, participants' overall adherence was $1.6 \%$ higher for each one-visit increase in total visits attended. Therefore, pervisit measures gave a better reflection of average and optimal adherence than per-participant measures.

Impact of Assumptions Made About Missing Data and Over-Adherence on Summary Measures

Assumptions made about how to handle intervals with unknown adherence (excluding or replacing as low adherence) had a larger impact on measures of optimal adherence than on measures of average adherence. Since optimal adherence is based on the proportion of data above a fixed cut-off $(\geq 90 \%)$, decisions about the unknown intervals affect the denominator but not the numerator. For example, when unknown adherence was assumed to be low ( $\leq 70 \%)$, only $48 \%$ of person-years in Mwanza had adherence $\geq 90 \%$, compared with $61 \%$ if unknown intervals were excluded from the calculation. In contrast, median per-visit adherence in Mwanza was similar if we assumed that adherence was low in unknown intervals or if we excluded these intervals (90 vs $95 \%$ respectively).

The relative difference between optimal adherence obtained by the two methods of handling unknown intervals increased with the amount of missing data. Mwanza had a higher proportion of visits with unknown adherence than HPTN 039 (21 vs $15 \%$ ); therefore, the choice of method for handling unknown intervals had a larger impact on calculations of optimal adherence in Mwanza.

When we assumed adherence was low $(\leq 70 \%)$ in unknown intervals, most measures of optimal adherence, and median per-visit adherence, were not changed by the value assigned to the unknown intervals. In contrast, overall adherence, and median per-participant adherence, were sensitive to the value assigned, and the relative difference between the two methods of handling unknown intervals became larger as lower values were used. The proportion of participants with adherence $\geq 90 \%$ was most sensitive to the value assigned to unknown intervals, with only $45 \%$ of participants in HPTN 039 having optimal adherence if we assumed adherence was $50 \%$ in unknown intervals, versus $54 \%$ if we assumed adherence was $70 \%$ in unknown intervals. At the other extreme, if we assumed adherence to be $100 \%$ in unknown intervals, then $74 \%$ of participants had optimal adherence. Thus, the proportion of visits with missing data and assumptions about adherence in those intervals can have a large influence on adherence estimates.

Relationship Between Calculated Adherence and Biologic Measures of Study Product

In Mwanza, genital HSV-2 DNA shedding was detected at $176(8 \%)$ of 2,198 visits. In HPTN 039, HSV-2 PCR positive GUD was detected at 891 (5\%) of 18,945 visits. The association of acyclovir with reduction in genital HSV-2 shedding (Mwanza) and incident GUD (HPTN 039) was greater among participants with calculated adherence 90-105\% in the preceding interval, than among participants with calculated adherence $<90 \%$ or $>105 \%$, or participants in whom adherence could not be calculated because tablets were not returned (Fig. 3).

\section{Discussion}

Interpretation of efficacy in trials of user-dependent HIV prevention strategies requires clear and consistent methods for measuring and reporting adherence to study product. Our results show that calculated adherence based on pill counts varies substantially, depending on the assumptions made about how to handle missing and inaccurate data and the choice of summary measure, and can provide substantially different impressions of product use. Recent HIV prevention trials have reported adherence to study product of $90 \%$ or higher based on pill counts $[3,4,7]$. However, such estimates may provide a simplistic or overly high indicator of adherence. Our analysis depicts a considerably more nuanced picture of adherence and emphasizes the need for more standardised approaches to reporting adherence data in order to facilitate interpretation of trial results and comparisons across trials of similar interventions.

Both HPTN 039 and Mwanza reported median adherence $>90 \%$ in their main trial publications [21, 22], but used different assumptions in their calculations so the measures were not comparable. We found that, when calculated in the same way with the same defined exclusion periods, median and overall adherence were very similar in both trials. However, measures of optimal adherence were substantially higher in HPTN 039, suggesting much higher levels of actual product use. The lack of an overall effect on biological markers of genital herpes reactivation, as reported in Mwanza [21], despite very high median adherence, shows that median adherence may not provide a very useful indicator of total study drug exposure during a trial. Summaries of optimal adherence, such as the proportion of person-years with adherence $\geq 90 \%$, may give a better reflection of actual product use, but are more sensitive to assumptions made about intervals with unknown 

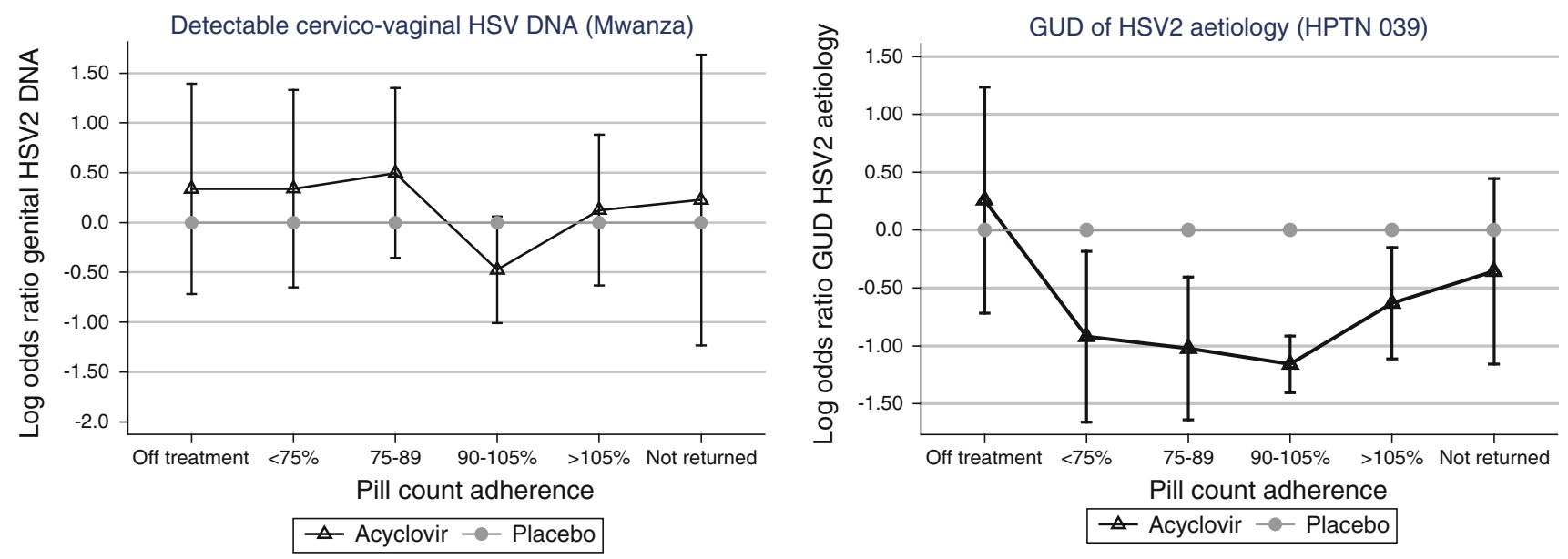

\begin{tabular}{|c|c|c|c|c|}
\hline \multirow{3}{*}{$\begin{array}{l}\text { Pill count } \\
\text { adherence } \\
\text { category }\end{array}$} & \multicolumn{2}{|c|}{ Mwanza } & \multicolumn{2}{|c|}{ HPTN 039} \\
\hline & \multicolumn{2}{|c|}{ N with HSV - 2 DNA / total (\%) } & \multicolumn{2}{|c|}{ N with HSV - 2 GUD / total (\%) } \\
\hline & Acyclovir & Placebo & Acyclovir & Placebo \\
\hline Off treatment $^{a}$ & $11 / 114(9.6 \%)$ & $7 / 98(7.1 \%)$ & $14 / 175(8.0 \%)$ & $13 / 182(7.1 \%)$ \\
\hline$<75 \%$ & $11 / 90(12.2 \%)$ & $9 / 102(8.8 \%)$ & $13 / 525(2.5 \%)$ & $31 / 536(5.8 \%)$ \\
\hline$<75-89 \%$ & $15 / 158(9.5 \%)$ & $11 / 181(6.1 \%)$ & $17 / 821(2.1 \%)$ & 53 / 935 (5.7 \%) \\
\hline $90-105 \%$ & 27 / $518(5.2 \%)$ & 40 / $493(8.1 \%)$ & 149 / $6199(2.4 \%)$ & 463 / $6598(7.0 \%)$ \\
\hline$>105 \%$ & 18 / $204(8.8 \%)$ & $15 / 191(7.9 \%)$ & $39 / 1018(3.7 \%)$ & $68 / 1051(6.5 \%)$ \\
\hline Not returned & $7 / 27$ (25.9\%) & $5 / 22(22.7 \%)$ & 14 / $463(3.0 \%)$ & 18 / $442(4.1 \%)$ \\
\hline
\end{tabular}

a Intervals when drug was not dispensed, including treatment interruptions for pregnancy

Fig. 3 Log OR and $95 \%$ confidence interval of genital HSV-2 DNA shedding (Mwanza, left) or in genital ulcer disease (GUD) of HSV-2 aetiology (HPTN 039, right), comparing acyclovir with placebo, within each pill count adherence category

adherence, particularly when the amount of missing data is large. Another disadvantage of measures of optimal adherence is that they dichotomise participants based on a fixed threshold value believed to represent sufficient drug exposure to achieve a therapeutic result; however, in practice, there are a range of factors that determine the threshold that is needed for a satisfactory treatment effect. Furthermore, as a review of adherence measurement points out, adherence in the $90-100 \%$ range is generally considered 'good', but this figure is not inconsistent with multi-day lapses in dosing during which drug concentrations fall below therapeutic levels, particularly with drugs with a short half-life like acyclovir [25].

Adherence in a particular interval can be unknown for several reasons: the participant may fail to return the tablets (missing data), calculated adherence may be $>100 \%$, or the tablets may be returned late, so a participant is issued with a new set of tablets before returning the first set. In Mwanza, we could not link tablets to the visit dispensed, so we assumed that all tablets returned were issued at the previous visit. In HPTN 039, a unique bottle identification number was recorded when tablets were dispensed and returned, so we were able to match the bottles to the visit dispensed.
Thus, unique study product identifiers may offer important advantages for assessing the 'measurability' of adherence in a trial. In addition, shorter times between visits may help reduce errors by reducing the amount of surplus tablets issued and allowing adherence messages to be reinforced more frequently. Mwanza had longer intervals between visits and was of longer duration than HPTN 039, which may have resulted in more intervals with unknown adherence. The proportion of visits with unknown adherence is a key factor influencing adherence estimates, and should be a greater focus in implementation of future trials and critiqued carefully when trials report their results.

In both trials, although the biological markers of genital herpes reactivation were different (PCR confirmed GUD in HPTN 039 and HSV-2 shedding ascertained at 3 time points in Mwanza), we found that the effect of acyclovir on biologic measures of anti-HSV-2 activity was similar among participants with pill counts that suggested adherence $>105 \%$ and those with adherence $<90 \%$, and lower than those with $90-105 \%$ adherence. This is consistent with recently-reported findings from the Partners in Prevention HSV/HIV Transmission Study, in which low and apparent over-adherence to acyclovir in HIV/HSV-2 dually 
infected persons was associated with a reduced effect on HIV-1 plasma viral load and HSV-2 GUD [26]. Acyclovir suppression significantly but incompletely reduces symptomatic genital ulcers and HSV-2 shedding, so both are imperfect markers of adherence and drug exposure [27]. However, since clinical effects such as genital ulcers are determined by adherence over a period of time, the incidence of GUD in HPTN 039 and HSV-2 shedding in Mwanza may provide a clinical measure of acyclovir exposure in these trials. Our findings suggest that participants with tablet counts indicating over-adherence are not actually taking the tablets; similar findings have been reported from studies of hypertension [28] and anti-obesity medication [29], and a study that used phenobarbital as a chemical marker of adherence [30]. Thus, when calculating summary measures, it may be better to assume that intervals with apparent over-adherence represent periods of low adherence, rather than excluding them or considering them as if they reflect $100 \%$ adherence. Reporting the proportion of visits with apparent over-adherence may provide important information about actual product use.

Although tablet counts suggesting over-adherence beyond a certain cut-off (e.g., $105 \%$ ) could represent pill dumping by participants to improve their apparent adherence, some shortfall in the number of tablets returned should be allowed, to account for the occasional dropped tablet or imprecision in accounting for tablets that were taken on the day dispensed and returned. There could also be some genuine overadherence, for example, if the participant forgets she or he has taken a tablet and takes an extra one. Using a fixed number of tablets to define over-adherence, rather than a percentage, could be a more reasonable approach, particularly with longer intervals between visits.

In addition to potential bias from intervals with unknown adherence, the choice of summary measure used to report trial adherence can provide a misleading estimate of product use. As our results demonstrate, reporting the median, either per-visit or per-participant, to summarise trial adherence can obscure a large number of subjects with poor adherence. In both trials, participants who attended more visits were in the trial for longer and had better adherence than those who attended less frequently. Thus summary measures that gave equal weight to the participants who dropped out early and those who remained in the trial provided an impression of lower adherence than measures that were weighted by the number of visits attended. This further highlights the need for a standardisation in calculating and reporting summary measures.

In both trials, we found that adherence based on selfreport of number of missed doses was higher than that based on pill counts. We did not use self-report in our adherence calculations for this paper; however, self-report was used to supplement the pill counts in the adherence calculations for the main trial papers. The tendency of selfreport to overestimate adherence, when compared with pill counts [3, 6], electronic monitoring devices [31] or drug detection [2, 6, 32] methods has been cited by other studies. Although asking participants about the number of missed pills may be of limited utility, other methods of self-evaluation, such as qualitative rating scales, may provide more reliable information [33].

Strengths of our study include analyses of data from two large HIV prevention efficacy trials of acyclovir suppression, with the same intervention but different visit schedules and different procedures for performing tablet counts. This allowed us to examine the impact of different study procedures and analysis assumptions on reported measures of adherence. Limitations of our study include that pill counts of returned study product are an imperfect method of assessing adherence, and did not allow us to assess finer patterns of adherence, for example, as would be possible with electronic daily monitoring. A further limitation is that clinic-based pill counts have been found to overestimate adherence compared with electronic monitoring [34, 35] or drug levels [6], and are subject to manipulation, as participants may discard unused pills to create an illusion of good adherence. The validity of pill counts to assess adherence relies on the assumption that unreturned tablets were ingested by the participant; however, estimates of adherence may be severely biased if unreturned tablets do not reflect actual product use. An additional limitation is that pill counts were done at every visit; however, GUD and HSV-2 shedding were measured less frequently. Although we had some clinical indicators of adherence, the half-life of acyclovir is $\sim 3 \mathrm{~h}$ with minimal accumulation [36], thus limiting acyclovir drug levels as a gold standard for evaluating our adherence measures using pill counts. Lastly, the Mwanza trial and the majority of the HPTN 039 sites were in resource-limited settings; therefore, our finding that pill counts suggestive of over-adherence are likely to represent pill dumping may not be generalisable to other settings.

Our results have demonstrated that there is a clear need for standardisation in adherence reporting. The CONSORT statement was developed to provide a set of guidelines for the clear, complete and transparent reporting of results from randomised controlled trials [37-39]. Although these guidelines implicitly recognise the importance of adherence in interpreting trial results, there are no recommendations for how to report adherence. For clinic-based pill counts of unused study product, authors should report the proportion of visits in which tablets were not returned, or where pill counts found adherence to be $>100 \%$, and how intervals with unknown adherence were handled in calculation of summary measures. To provide a measure of uncertainty of these adherence metrics, it is useful to report the summary measures using different assumptions 
(e.g. excluding unknown intervals and considering these as low adherence). We recommend that trials should report measures of study medication adherence coverage (i.e. including missed visits and time off treatment), as the most relevant measure for understanding results from the intentto-treat analysis. Although all summary measures provide an imperfect assessment of adherence, overall adherence, since it takes into account the total days that each participant is in the trial, may provide a reasonable reflection of drug exposure on average over the duration of the trial. Some estimate of the amount of person-time with optimal adherence may be useful, since this relates exposure to levels needed to achieve a biological effect. However, in addition to the problems of dichotomising adherence as discussed above, there can be difficulties in operationalising 'optimal' adherence: the definition is likely to differ between study drugs, formulations and dosing regimens (and may not be known for new investigational products), creating further complications for standardising definitions across trials. Summary measures of median adherence, either per-visit or per-participant, are not particularly informative.

In summary, we have shown that summary measures of adherence in two recent HIV prevention trials of acyclovir varied considerably depending on assumptions and analysis method. Our findings of a relationship between adherence and clinical markers (GUD or HSV-2 genital shedding) indicate that intervals of apparent over-adherence are likely to represent pill-dumping. Thus, the proportion of visits with unknown adherence, either because tablets are not returned or calculated adherence is too high, may provide important information. The impact of the assumptions made on calculated measures indicate the important need for standardisation of best practices in adherence reporting, to aid in the interpretation and understanding of userdependent HIV prevention interventions.

Acknowledgments We thank the participants in both trials, and the HPTN 039 and Mwanza trial teams at the study sites, for their contributions to this study. The HPTN 039 trial was supported through funding from the United States National Institutes of Health (U01 AI052054 and AI30731) and by the HIV Prevention Trials Network (HPTN) under Cooperative Agreement \#U01 AI46749, sponsored by the National Institute of Allergy and Infectious Diseases, National Institute of Child Health and Human Development, National Institute of Drug Abuse, National Institute of Mental Health, and Office of AIDS Research NIH UO1 AI52054-06. The Mwanza trial was supported by grants from the Wellcome Trust (066688), the United Kingdom Medical Research Council (70988), and the United Kingdom Department of International Development. Additional funding support for the current study was provided by R01-MH095507.

Open Access This article is distributed under the terms of the Creative Commons Attribution License which permits any use, distribution, and reproduction in any medium, provided the original author(s) and the source are credited.

\section{References}

1. Abdool Karim Q, Abdool Karim SS, Frohlich JA, et al. Effectiveness and safety of tenofovir gel, an antiretroviral microbicide, for the prevention of HIV infection in women. Science. 2010;329(5996):1168-74.

2. Marrazzo J, Ramjee G, Nair G, et al. Pre-exposure prophylaxis for HIV in women: daily oral tenofovir, oral tenofovir/emtricitabine or vaginal tenofovir gel in the VOICE study. In 20th conference on retrovirus and opportunistic infections, Atlanta, Georgia; 2013.

3. Grant RM, Lama JR, Anderson PL, et al. Preexposure chemoprophylaxis for HIV prevention in men who have sex with men. N Engl J Med. 2010;363(27):2587-99.

4. Baeten JM, Donnell D, Ndase P, et al. Antiretroviral prophylaxis for HIV prevention in heterosexual men and women. N Engl J Med. 2012;367(5):399-410.

5. Thigpen MC, Kebaabetswe PM, Paxton LA, et al. Antiretroviral preexposure prophylaxis for heterosexual HIV transmission in Botswana. N Engl J Med. 2012;367(5):423-34.

6. Van Damme L, Corneli A, Ahmed K, et al. Preexposure prophylaxis for HIV infection among African women. N Engl J Med. 2012;367(5):411-22.

7. Celum C, Wald A, Lingappa JR, et al. Acyclovir and transmission of HIV-1 from persons infected with HIV-1 and HSV-2. N Engl J Med. 2010;362(5):427-39.

8. Lagakos S, Gable A, editors. Methodological challenges in biomedical HIV prevention trials. Washington DC: The National Academics Press; 2008.

9. Masse B, Boily MC, Dimitrov D, Desai K. Efficacy dilution in randomized placebo-controlled vaginal microbicide trials. Emerg Themes Epidemiol. 2009;6:5.

10. Amico K. Adherence to preexposure chemoprophylaxis: the behaviour bridge from efficacy to effectiveness. Curr Opin HIV AIDS. 2012;7(6):542-8.

11. Karim SS, Kashuba AD, Werner L, Karim QA. Drug concentrations after topical and oral antiretroviral pre-exposure prophylaxis: implications for HIV prevention in women. Lancet. 2011;378(9787):279-81.

12. Mugwanya K, Baeten JM, Mugo NR, Irungu E, Ngure K, Celum C. High-dose valacyclovir HSV-2 suppression results in greater reduction in plasma HIV-1 levels compared with standard dose acyclovir among HIV-1/HSV-2 coinfected persons: a randomized, crossover trial. J Infect Dis. 2011;204(12):1912-7.

13. Microbicide Trials Network (MTN). Microbicide trials network MTN-003 (VOICE) study protocol. 2010. http://www.mtnstopshiv. org/sites/default/files/attachments/MTN-003_FINAL_Version_2.0 _31DEC2010.pdf. Accessed Nov 2012.

14. Pool R, Montgomery CM, Morar NS, et al. A mixed methods and triangulation model for increasing the accuracy of adherence and sexual behaviour data: the Microbicides Development Programme. PLoS ONE. 2010;5(7):e11600.

15. Muchomba FM, Gearing RE, Simoni JM, El-Bassel N. State of the science of adherence in pre-exposure prophylaxis and microbicide trials. J Acquir Immune Defic Syndr. 2012;61(4):490-8.

16. van der Straten A, Van Damme L, Haberer JE, Bangsberg DR. Unraveling the divergent results of pre-exposure prophylaxis trials for HIV prevention. AIDS. 2012;26(7):F13-9.

17. Berg KM, Arnsten JH. Practical and conceptual challenges in measuring antiretroviral adherence. J Acquir Immune Defic Syndr. 2006;43(Suppl 1):S79-87.

18. Tolley EE, Harrison PF, Goetghebeur E, et al. Adherence and its measurement in phase $2 / 3$ microbicide trials. AIDS Behav. 2010;14(5):1124-36. 
19. Blaschke TF, Osterberg L, Vrijens B, Urquhart J. Adherence to medications: insights arising from studies on the unreliable link between prescribed and actual drug dosing histories. Annu Rev Pharmacol Toxicol. 2012;52:275-301.

20. van der Straten A, Montgomery ET, Hartmann M, Minnis A. Methodological lessons from clinical trials and the future of microbicides research. Curr HIV/AIDS Rep. 2013;10:89-102.

21. Watson-Jones D, Weiss HA, Rusizoka M, et al. Effect of herpes simplex suppression on incidence of HIV among women in Tanzania. N Engl J Med. 2008;358(15):1560-71.

22. Celum C, Wald A, Hughes J, et al. Effect of aciclovir on HIV-1 acquisition in herpes simplex virus 2 seropositive women and men who have sex with men: a randomised, double-blind, placebo-controlled trial. Lancet. 2008;371(9630):2109-19.

23. Jacob ST, Baeten JM, Hughes JP, et al. A post-trial assessment of factors influencing study drug adherence in a randomized biomedical HIV-1 prevention trial. AIDS Behav. 2011;15(5):897-904.

24. Watson-Jones D, Baisley K, Rusizoka M, et al. Measurement and predictors of adherence in a trial of HSV suppressive therapy in Tanzania. Contemp Clin Trials. 2009;30(6):504-12.

25. Vrijens B, Urquhart J. Patient adherence to prescribed antimicrobial drug dosing regiments. J Antimicrob Chemother. 2005; 55:616-27.

26. Donnell DJ, Baeten JM, Hong T, et al. Correlation between pill counts and biologic effects in an HIV-1 prevention clinical trial: implications for measuring adherence. AIDS Behav. 2012;17(2): 632-9.

27. Gupta R, Wald A. Genital herpes: antiviral therapy for symptom relief and prevention of transmission. Expert Opin Pharmacother. 2006;7(6):665-75.

28. Rudd P, Byyny RL, Zachary V, et al. The natural history of medication compliance in a drug trial: limitations of pill counts. Clin Pharmacol Ther. 1989;46:169-76.

29. Pocock SJ, Abdalla M. The hope and hazards of using compliance data in randomised controlled trials. Stat Med. 1998;17: 303-17.
30. Pullar T, Kumar S, Tindall H, Feely M. Time to stop counting the tablets? Clin Pharmacol Ther. 1989;46:163-8.

31. Wilson IB, Carter AE, Berg KM. Improving the self-report of HIV antiretroviral medication adherence: is the glass half full or half empty? Curr HIV/AIDS Rep. 2009;6:177-86.

32. Minnis AM, Gandham S, Richardson BA, Guddera V, et al. Adherence and acceptability in MTN 001: a randomised crossover trial of daily oral and topical tenofovir for HIV prevention in women. AIDS Behav. 2013;17:737-47.

33. Lu M, Safren SA, Skolnik P, et al. Opitmal recall period and response taks for self-reported HIV medication adherence. AIDS Behav. 2008;12:86-94.

34. Liu H, Golin CE, Miller LG, et al. A comparison study of multiple measures of adherence to HIV protease inhibitors. Ann Intern Med. 2001;134:968-77.

35. Jasti S, Siega-Riz AM, Cogswell ME, Hartzema AG. Correction for errors in measuring adherence to prenatal multivitamin/mineral supplement use among low-income women. J Nutr. 2006; 136:479-83.

36. Lu Y, Celum C, Wald A, et al. Acyclovir achieves a lower concentration in African HIV-seronegative, herpes simplex virus 2 -seropositive women than in non-African populations. Antimicrob Agents Chemother. 2012;56(5):2777-9.

37. Moher D, Hopewell S, Schulz KF, et al. CONSORT 2010 explanation and elaboration: updated guidelines for reporting parallel group randomised trials. J Clin Epidemiol. 2010;63(8): e1-37.

38. Schulz KF, Altman DG, Moher D. CONSORT 2010 statement: updated guidelines for reporting parallel group randomised trials. BMJ. 2010;340:c332.

39. Begg $\mathrm{C}$, Cho $\mathrm{M}$, Eastwood $\mathrm{S}$, et al. Improving the quality of reporting of randomized controlled trials. The CONSORT statement. JAMA J Am Med Assoc. 1996;276(8):637-9. 